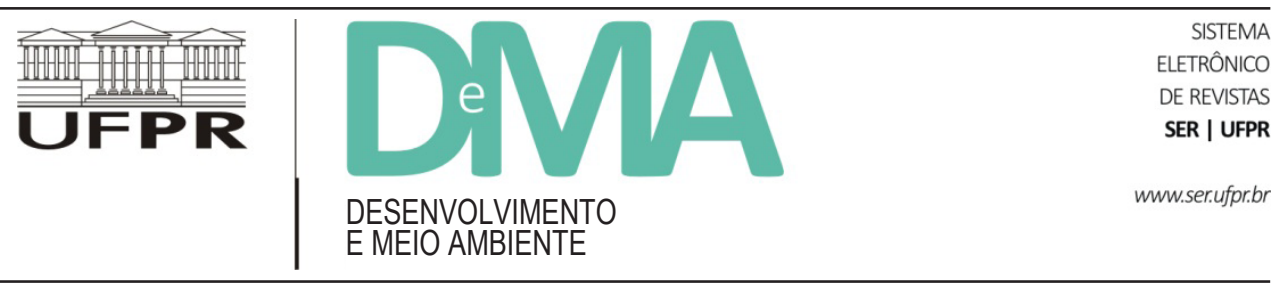

\title{
Una investigación participativa en la costa uruguaya: características, desafíos y oportunidades para la enseñanza universitaria
}

\section{Uma pesquisa participativa na costa uruguaia: características, desafios e oportunidades para o ensino universitário}

\section{A Participatory Research in Coastal Uruguay: Characteristics, Challenges and Opportunities for University Education}

\author{
Micaela TRIMBLE ${ }^{1,2^{*}}$, Patricia IRIBARNE ${ }^{3}$, Marila LÁZARO ${ }^{4}$ \\ ${ }^{1}$ Natural Resources Institute, University of Manitoba, Winnipeg, Canada. \\ ${ }^{2}$ Centro de Estudos do Mar (CEM), Universidade Federal do Paraná (UFPR), Pontal do Sul, PR, Brasil. \\ ${ }^{3}$ Licenciatura en Biología Humana, Universidad de la República, Montevideo, Uruguay. \\ ${ }^{4}$ Unidad de Ciencia y Desarrollo, Facultad de Ciencias, Universidad de la República, Montevideo, Uruguay. \\ *E-mail de contacto: mica.trimble@gmail.com
}

Artículo recibido el 10 de marzo, 2014, versión final aceptada el 12 de septiembre, 2014.

RESUMEN Los enfoques que promueven algún tipo de participación comunitaria están siendo cada vez más utilizados para abordar problemáticas socio-ambientales. Uno de éstos son las investigaciones participativas (IP): estrategias de investigación en las que diversos actores trabajan de forma colaborativa en relación a un tema de interés común. El grupo generado para llevar adelante la investigación participa de forma horizontal en cada una de las etapas, desde elegir la pregunta de investigación hasta la difusión de los resultados. La aplicación de estos enfoques en la formación universitaria representa un desafío desde varios puntos de vista: resignifica el rol del especialista; cuestiona las visiones tradicionales de ciencia y su método; y promueve la reflexión, no solo sobre estas temáticas, sino también sobre el binomio investigación/acción. Tras caracterizar el enfoque específico de las IP, el objetivo de este trabajo es describir tres experiencias de formación universitaria vinculadas a una IP en la pesquería artesanal de Piriápolis (Uruguay), y a partir de ellas discutir algunas potencialidades y desafíos de la inclusión de este enfoque en la enseñanza universitaria en Uruguay. La IP en Piriápolis fue iniciada en 2011 con la meta de abordar problemáticas locales vinculadas a la pesca artesanal entre distintos actores implicados: pescadores artesanales, investigadores universitarios, organización gubernamental a cargo del manejo pesquero y ONGs. Esta IP fue iniciada en el marco de una investigación doctoral, dio lugar a una tesina de grado, y sirvió de plataforma para un curso de grado de ciencias. De estas experiencias se desprende que las IP constituyen enfoques transdisciplinarios que potencian metodológica y conceptualmente las iniciativas de 
formación universitaria integral con un énfasis en la inter o transdisciplina y el diálogo de saberes. Asimismo, las IP pueden ser compatibles con la realización de tesis de grado y posgrado, y pueden ser vinculadas a diversas plataformas educativas.

Palabras claves: investigación participativa; universidad; pesca artesanal.

RESUMO Enfoques que promovem algum tipo de participação comunitária estão sendo cada vez mais utilizados para abordar problemáticas socioambientais. Um exemplo são as pesquisas participativas (PP): estratégias de pesquisa nas quais diversos atores trabalham de forma colaborativa para um tema de interesse comum. $\mathrm{O}$ grupo de trabalho formado para conduzir a pesquisa participa, de forma horizontal, em todas as etapas, desde a definição da pergunta de pesquisa até a difusão dos resultados. A aplicação destes enfoques na formação universitária representa desafios por vários pontos de vista: redefine o papel do especialista; questiona as visões científicas tradicionais e seus métodos; e promove a reflexão, não só sobre estas temáticas, mas também sobre o binômio pesquisa/ação. Após caracterizar o enfoque específico das PP, o objetivo desde trabalho é descrever três experiências de formação universitária vinculadas a uma PP na pesca artesanal de Piriápolis (Uruguai) e, a partir destas experiências, discutir potencialidades e desafios da inclusão deste enfoque no ensino universitário no Uruguai. A PP em Piriápolis foi iniciada em 2011 visando abordar problemáticas locais vinculadas à pesca artesanal entre distintos atores: pescadores artesanais, pesquisadores universitários, organização governamental encarregada da gestão pesqueira e ONGs. Esta PP foi iniciada durante uma pesquisa de doutorado, permitiu uma dissertação de graduação e serviu de plataforma para uma disciplina de graduação em ciências. Destas experiências, conclui-se que as PP constituem enfoques transdisciplinares que potenciam, metodológica e conceitualmente, iniciativas universitárias integradas com ênfase na inter ou transdisciplinaridade e no diálogo entre saberes. Ademais, as PP são compatíveis com a realização de estudos e trabalhos de graduação e pós-graduação e podem ser vinculadas a diversas plataformas educativas.

Palavras-chave: pesquisa participativa; universidade; pesca artesanal.

ABSTRACT Approaches that promote some kind of community participation have been increasingly used for addressing socio-environmental problems. One of these approaches is participatory research (PR), a research strategy in which diverse stakeholders work collaboratively around an issue of common interest. The group formed to carry out the research participates horizontally in every stage, from choosing the research question to the dissemination of the results. The application of this approach in university education represents a challenge from various perspectives: it redefines the role of the specialist; it questions the traditional views of science and its method; and it promotes the reflection, not only about these topics but about the research/action binomial. After characterizing the participatory research approach, the objective of this paper is to describe three experiences of university education related to a participatory research in the artisanal fishery of Piriápolis (Uruguay). Based on them we discuss the potentials and challenges of the inclusion of this approach in university education in Uruguay. The PR in Piriápolis was initiated in 2011 aiming at addressing local problems of the artisanal fishery among different stakeholders: artisanal fishers, university researchers, government agency in charge of fisheries management, and NGOs. This PR was initiated during a doctoral research, encompassed an undergraduate research, and served as a platform for an undergraduate course in sciences. From these experiences we argue that PR represents a transdisciplinary approach that strengthens, methodologically and conceptually, university initiatives for integral education with emphasis on inter or transdisciplinarity and knowledge dialogue. Additionally, PR can be compatible with undergraduate and graduate theses, and can be linked to diverse educational platforms.

Keywords: participatory research; university; artisanal fisheries. 


\section{Introducción}

Si a la imagen contemporánea de los sistemas naturales, que los presenta como redes complejas de elementos interdependientes, con cierto grado de plasticidad e indeterminación (lo que los hace difícilmente predecibles) (Holling, 1987), le sumamos su imbricación o interrelación con los sistemas sociales (imbricación que recoge el concepto de "ambiente"), debemos reconocer que tratamos con sistemas complejos que generan incertidumbre sobre beneficios, riesgos, y consecuencias de cualquier intervención. A esto debemos sumar la presencia de diversidad de actores y por tanto diversidad de intereses, percepciones, conocimientos y creencias entretejidas a cierta temática. Es así que en las últimas tres décadas se ha defendido y justificado (desde puntos de vistas éticos y epistemológicos) no solo el involucramiento de distintas disciplinas académicas a la hora de diagnosticar y solucionar los problemas complejos sino también el de las comunidades implicadas, asociadas al problema o situación particular (Lázaro et al., 2013).

La metodología científica ha ido acomodándose a esta nueva imagen de naturaleza y también a una nueva imagen del rol y alcances de la propia ciencia. Los enfoques inter y transdisciplinarios, enfatizando en la cooperación y diálogo entre disciplinas y metodologías, plantean una forma de lidiar con problemas complejos que requiere no solo de múltiples miradas sino de su entrecruzamiento. Estas nuevas formas de enfrentar problemas complejos no suprimen las formaciones disciplinarias ni las especializaciones sino que parten de ellas y las invitan a mantenerse abiertas, en relación y diálogo con otras formas de conocimiento. A pesar de sus variadas definiciones (ver por ej., Klein, 1990; Ander-Egg, 1994; de Sousa Santos, 2010), la diferencia entre inter y transdisciplina puede situarse en el hecho de que los enfoques transdisciplinarios consideran también saberes no académicos. Esta caracterización hace que dichos enfoques sean más comunes en áreas vinculadas con el ambiente, en donde la multiplicidad de actores implicados es proverbial. La transdisciplina puede entenderse como la formulación de problemas, y de propuestas para comprenderlos y resolverlos, mediante la interacción de especialistas de diversas disciplinas y de actores que, aún sin provenir de disciplinas académicas, poseen experiencia y conocimiento relevantes en relación al problema (Olivé, 2011; Lang et al., 2012).

Pero abrir las puertas de una investigación científica a otros actores no "disciplinados" es un reto para la ciencia. Existen tres argumentos principales que recogen los distintos discursos que apoyan la adopción de enfoques participativos tanto para la gestión política de la ciencia y tecnología como para la generación de conocimiento (Fiorino, 1990; Blackstock et al., 2007). El argumento sustantivo propone que el juicio, la experiencia y el conocimiento de los ciudadanos no expertos, pueden ser tan pertinentes como los de los expertos en la identificación de problemas y soluciones que a veces los expertos no vislumbran. Por su parte, el argumento instrumental plantea que la participación de los no expertos en una investigación, legitima el proceso y lleva a resultados que son aceptados como válidos por todos los participantes. Por último, el argumento normativo plantea que la participación pública es compatible con valores democráticos y éticos ya que los ciudadanos tienen derecho a participar en los asuntos y decisiones que los afecten.

Estos argumentos explican la creciente aplicación de investigaciones participativas en el contexto de conflictos ambientales y de manejo de recursos naturales, incluyendo pesquerías (Brzeski et al., 2001; Cambel \& Salagrama, 2001; Conway \& Pomeroy, 2006; Gonsalves et al., 2006; Hartley \& Robertson, 2006; Wilmsen et al., 2008; Wiber et al., 2009; Shirk et al., 2012). Una investigación participativa (IP) es un enfoque de investigación en el cual los investigadores trabajan de forma cooperativa o colaborativa con integrantes de la comunidad (y a veces otros actores externos a ella) implicados en una problemática. Los diferentes actores participan de cada una de las etapas como co-investigadores, desde elegir la pregunta de investigación hasta la difusión de los resultados (Cornwall \& Jewkes, 1995). Además de la co-producción de conocimiento, esta estrategia permite abordar o resolver problemas locales, articulando y potenciando los saberes académicos con los locales (Fals Borda, 1987). Cabe destacar que así como la inter y transdisciplina no intentan sustituir a la formación disciplinar, las IP no pretenden ni podrían sustituir a las investigaciones convencionales. Dependiendo de los problemas a tratar, y de los contextos en cada caso, es que se podrán o no desplegar diversas 
estrategias y grados de participación pública en ciencia y tecnología.

En el caso específico de las pesquerías, la creciente aplicación de investigaciones participativas desde los años 2000 (también conocidas como investigaciones pesqueras cooperativas), y/o de investigaciones con cierto grado de participación local, está vinculada con la prevalencia de relaciones conflictivas entre pescadores, gestores e investigadores, lo cual dificulta el manejo o gestión de los recursos pesqueros (Kaplan \& McCay, 2004; Wiber et al., 2004; 2009; Conway \& Pomeroy, 2006; Hartley \& Robertson, 2006; Johnson \& van Densen, 2007; Jacobsen et al., 2012). A pesar de su creciente aplicación, las investigaciones participativas en pesquerías, al igual que en otros contextos ambientales, han enfrentado varias dificultades, incluyendo, entre otros, el poco tiempo disponible por parte de los pescadores, su desconfianza sobre el destino final de los resultados de la investigación, y su escaso empoderamiento; menos oportunidades de publicación para los académicos; y la escasa participación de otros actores, como gestores y organizaciones para la conservación (Wiber et al., 2004; Conway \& Pomeroy, 2006; Hartley \& Robertson, 2006). Uruguay no se escapa de las dificultades para desarrollar investigaciones participativas. Como se ha señalado en otros contextos (Wiber et al., 2004), es frecuente encontrar que los pescadores se convierten en sujetos de estudio o asistentes de campo en investigaciones llevadas adelante por investigadores de ramas naturales y sociales de la ciencia.

El propósito de este artículo es describir tres experiencias de formación universitaria vinculadas a una IP que se desarrolla en la pesquería artesanal de Piriápolis (Uruguay) desde 2011: un curso de grado, una tesina de grado y una tesis de doctorado (la última fue la que dio origen a la IP). A partir de estas tres experiencias discutimos algunas oportunidades y potencialidades de las investigaciones participativas para la formación universitaria en general y como herramienta para la formación integral en Uruguay, entendiendo ésta como la articulación de las tres funciones universitarias (investigación, enseñanza y extensión). El artículo se estructura en tres componentes. En primer lugar, se conceptualizan y caracterizan las investigaciones participativas, haciendo una pequeña síntesis histórica de su origen, y se señalan ciertos criterios claves para su desarrollo. En segundo lugar, se presenta la IP de Piriápolis en el contexto universitario en que tuvo lugar, describiendo las tres experiencias antes mencionadas y su rol en la formación de los estudiantes. Por último, a partir de estas experiencias, se discuten algunas oportunidades y desafíos para el desarrollo de investigaciones participativas en el ámbito universitario.

\section{Participación en investigación: origen, metas $y$ características}

\subsection{Origen y conceptualización}

A diferencia de las investigaciones convencionales (aquellas llevadas a cabo por académicos), en las IP se busca reducir la brecha entre investigadores e integrantes de la comunidad a partir de la oportunidad de compartir el poder y la responsabilidad para tomar decisiones a lo largo del proyecto que emprenden juntos (Cornwall \& Jewkes, 1995; Kemmis \& McTaggart, 2005). En relación a sus metas, las IP están diseñadas para involucrar a integrantes de una comunidad en el análisis de su realidad y encauzar la comprensión de los problemas y la discusión de posibles soluciones. Además de este empoderamiento de la comunidad, los resultados positivos de este enfoque incluyen una mayor credibilidad en el proceso de la investigación, mejora de las relaciones entre los actores participantes, aprendizaje mutuo, co-producción de conocimiento mediante diálogo de saberes, resolución de conflictos, y fortalecimiento de capacidades de los participantes (Cornwall \& Jewkes, 1995; Brzeski et al., 2001; Arnold \& Fernandez-Gimenez, 2007; Johnson, 2010; Trimble \& Berkes, 2013; Trimble \& Lázaro, 2014).

De la misma forma en que actualmente el uso de este enfoque participativo de investigación y acción política es encontrado en disciplinas y campos variados (por ej., sociología, antropología, psicología, salud, ciencias naturales, ciencias socio-ambientales, participación pública), el origen y desarrollo de las investigaciones participativas se vio nutrido por influencias diversas (ver Fisher, 2000; Chevalier \& Buckles, 2013). Entre las más destacables se encuentra la investigación - 
acción (Lewin, 1946) y la educación popular (Freire, 1970). También influyó de forma considerable la obra del colombiano Orlando Fals-Borda que universalizó las propuestas de Freire para una colección de casos de estudio y análisis teóricos sobre la investigación (acción) participativa en comunidades de todo el mundo (Fals-Borda \& Rahman, 1991). ${ }^{1}$

A partir de estas experiencias e influencias diversas, este tipo de investigaciones entre "expertos y no expertos" han ido adoptando diferentes denominaciones (aunque a veces con significados variados incluso dentro de una misma denominación): investigación participativa (IP), investigación acción participativa (IAP), investigación acción (IA), investigación de base comunitaria (IBC), investigación colaborativa (IC), entre otros. ${ }^{2}$ En el presente trabajo utilizamos el término "investigación participativa" con la definición desarrollada en los párrafos anteriores.

\subsection{Condiciones para desarrollar y evaluar una investigación participativa (IP)}

A partir de la literatura sobre investigaciones participativas (Reason, 1994; Balcazar, 2003; Kemmis \& McTaggart, 2005; Blackstock et al., 2007; Kindon, 2008) y sobre evaluación de mecanismos de participación pública (Rowe \& Frewer, 2000; Stephens \& Berner, 2011), podemos sugerir al menos nueve condiciones (interconectadas) para el desarrollo de este enfoque, las cuales también pueden ser utilizadas como criterios para su evaluación (Trimble \& Lázaro, 2014):

$\underline{1 .}$ Interés local y de los demás actores implicados en la problemática a abordar

Si se considera que una IP debe surgir a partir de los problemas e intereses de una comunidad, se vislumbran al menos dos posibilidades: que integrantes de la comunidad inviten a otros grupos de actores para trabajar de forma conjunta en el diagnóstico y abordaje de la problemática, o que algún actor externo perciba un problema local, realice el diagnóstico del mismo con la comunidad y juntos inicien una investigación o acción al respecto. En cualquier caso, se debe dar un interés doble, interés en un tema particular, e interés en abordarlo a través de una IP.

2. Participación de diversos grupos de actores implicados (stakeholders)

Una vez elegido el problema a tratar en la IP, se debe propiciar la participación o involucramiento de otros grupos que estén implicados en la temática desde sus distintos ángulos (académicos, organizaciones sociales, organizaciones gubernamentales, etc.). Enriquecer el tratamiento del problema mediante la conjugación de los enfoques y saberes de diferentes implicados que tienen contacto y/o experiencia en el tema (y no solo de los "expertos") es un elemento clave de la IP.

\section{Representatividad de los participantes}

Cuando la IP está vinculada a ámbitos de gestión política, es especialmente importante que los participantes sean representativos de los grupos de implicados a los que pertenecen. En un contexto más amplio, la representatividad de los participantes afectará a la legitimidad de los resultados o actuaciones del grupo formado alrededor de la IP. De forma de asegurar la representatividad de los actores locales, es recomendable conocer en una primera instancia las dinámicas y relaciones sociales en la comunidad.

\section{Involucramiento de los diversos grupos de actores en cada una de las etapas}

Todo el proceso de la IP debe ser desarrollado colectivamente, incluyendo la definición de objetivos concretos a perseguir, el diseño de la metodología, la planificación de las actividades a concretar y su evaluación. Este punto posiblemente sea el que marca una de las mayores diferencias entre las IP, y las investigaciones convencionales, o incluso aquellas investigaciones que

\footnotetext{
${ }^{1}$ En los años 90 este enfoque empezó a tener mayor presencia también en el hemisferio norte (Hall, 1992). En 1997 fue estimado que en al menos 2500 universidades en 61 países se enseñaba sobre, o se practicaban, investigaciones participativas (Fals Borda, 2006).

${ }^{2}$ Algunos autores consideran que la IP y la IAP constituyen dos escuelas que se originaron tras la influencia de Freire (Chambers, 1994; Cornwall \& Jewkes, 1995), mientras que otros señalan que la IP entra dentro de la IAP (Kemmis \& McTaggart, 2005).
} 
contemplan la participación de los implicados pero a partir de un objetivo definido solamente por los expertos.

\section{Facilitación}

Los procesos de diálogo entre diferentes actores implicados precisan de una figura, conocida como facilitador/a, que coordine los intercambios y contribuya al desarrollo de objetivos comunes. El papel del facilitador es fundamental para lograr que el diálogo de saberes se establezca de la forma más balanceada posible, sin jerarquías ni grupos de actores que lideren el desarrollo de la IP. Como algunos grupos de actores (expertos, actores gubernamentales) tendrán inherentemente más poder que otros (Gaventa \& Cornwall, 2001), el facilitador debe reconocerlo e invitar a los actores con menos poder (comunitarios) a participar de las discusiones. Propiciar la tolerancia y respeto mutuo entre participantes, el respeto por sus visiones, conocimientos y argumentos, el balance de las intervenciones, alimentará la toma de decisiones colectivas. Cuando existe tensión o desconfianza entre los grupos de actores participantes, se recomienda que el proceso sea facilitado por un actor no implicado en la temática tratada, de forma de asegurar un diálogo balanceado entre los diferentes conocimientos y perspectivas (Chevalier \& Buckles, 2013).

\section{Toma de decisiones colectivas tras instancias deli-} berativas

En una IP la toma de decisiones sobre el propio proceso de la investigación debe ser colectiva. Para que ello suceda, y vinculado con el manejo y análisis profundo de los argumentos y enfoques de cada grupo de participantes, es necesario que se propicien diálogos colectivos de evaluación de argumentos relevantes, y ponderación de los mismos. El arribo a consensos puede ser una de las estrategias para la toma de decisiones colectivas.

\section{Manejo apropiado de la información}

En una IP, lograr vías de información adecuada y de calidad depende de lograr que los principales implicados participen del proceso y disponibilicen sus conocimientos e información. Lo esperable es que esa información que se disponibilice sea adecuada y en cantidad y calidad acorde con lo necesario para la IP en cuestión. Esto implica lograr una buena comunicación que supere los problemas de los tecnicismos y jergas de los distintos grupos y la confianza en el proceso que genere una visión de grupo y objetivos compartidos. También se requiere que la información manejada sea balanceada entre grupos de interés y que se generen acuerdos sobre la validación de las fuentes.

\section{$\underline{\text { 8. Adaptabilidad durante ciclos de planificación, acción, }}$} observación y reflexión

La IP es un proceso iterativo de búsqueda de soluciones a problemas locales, que consta de varias etapas: planificación (identificación del problema, definición de preguntas de investigación); acción y observación (definición de metodologías, colecta de información y análisis), y reflexión (sobre la etapa anterior). Estas etapas sirven como guía más que como pasos estrictos, ya que de hecho, existe solapamiento entre ellas (Kemmis \& McTaggart, 2005). Estos ciclos deben cumplirse con la suficiente plasticidad o adaptabilidad como para que, a partir de la continua reflexividad sobre objetivos y resultados obtenidos, puedan surgir nuevas preguntas e intereses por parte del grupo.

\section{Costo (esfuerzo) - eficacia (logros)}

La relación entre los distintos tipos de posibles costos (económicos o vinculados a tiempos y esfuerzos personales) y la "eficacia" de la IP debe asegurar su continuidad. Además de los costos económicos, debe contemplarse la relación entre los esfuerzos que cada participante o grupo de actores realiza para formar parte de la IP, y su percepción sobre los logros que se obtienen por hacerlo (tanto individuales como colectivos).

\section{Investigación participativa en la pesquería artesanal de Piriápolis}

En marzo de 2011, las autoras iniciaron los primeros pasos hacia una IP en la pesquería artesanal de Piriápolis $^{3}$ (costa uruguaya del Río de la Plata), en el

\footnotetext{
${ }^{3}$ Por una descripción más detallada de esta pesquería ver Trimble (2013) o Trimble \& Johnson (2013).
} 
marco de la tesis doctoral de M.T. en la Universidad de Manitoba (Canadá). La etapa inicial consistió en relevar cuál era la problemática local que los pescadores artesanales consideraban como más prioritaria, la cual sería abordada en conjunto con otros actores. La problemática seleccionada por la mayoría de los pescadores entrevistados fue la interacción con los lobos y leones marinos (Arctocephalus australis y Otaria flavescens), que se alimentan de las presas en las artes de pesca (redes de enmalle y palangres) y ocasionan daños en las mismas. Luego procedimos a invitar a diferentes actores implicados (DINARA - Dirección Nacional de Recursos Acuáticos, investigadores del área pinnípedos y pesquerías, y ONGs locales que protegen a la fauna marina y/o buscan el desarrollo sustentable de Piriápolis) a ser parte de una IP con foco en dicha problemática.

Tras la aceptación de la invitación por parte de la mayoría de los convocados (por más información ver Trimble, 2013 o Trimble \& Lázaro, 2014), en mayo de 2011 tuvo lugar el primer taller entre los diferentes actores, quienes con el paso de los meses decidieron llamarse "Grupo POPA - Por la Pesca Artesanal en Piriápolis”. En 2011 POPA fue conformado por 7 pescadores, el encargado de la Unidad de Pesca Artesanal de la DINARA, 4 biólogos/ecólogos egresados y/o docentes de la Facultad de Ciencias - UdelaR, una investigadora del área de ciencias humanas de la Universidad Católica del Uruguay (realizando su maestría en comunicación y cultura), y dos representantes de organizaciones locales - Ecópolis y SOS Rescate de Fauna Marina. Las autoras constituían el equipo organizador y facilitador de la investigación participativa, estando a cargo de: (i) asegurar que los diferentes grupos de actores participaran de cada una de las etapas del proceso; (ii) invitar a más pescadores de los distintos sitios de desembarque en la zona de Piriápolis a participar, mantenerlos informados de los avances, y recabar sus aportes (por ej., sobre la comprensión de la problemática y posibles actuaciones al respecto); (iii) planificar y facilitar talleres (instancias colectivas de trabajo, ver a continuación); y (iv) propiciar otras instancias de intercambio de opiniones y toma de decisiones tanto en modalidad presencial (trabajo en subgrupos) como no presencial, utilizando diversos medios de comunicación (correo electrónico, celular y teléfono).
Durante los talleres (realizados en Piriápolis de forma mensual) se intercambiaban conocimientos, se discutían las estrategias para abordar los problemas o conflictos identificados, y se llegaba a acuerdos de trabajo. Los talleres tenían como objetivo constituirse en foros de aprendizaje colectivo en los cuales participaban personas con diferentes posiciones, conocimientos, experiencias e intereses (a veces opuestos). La figura del facilitador o moderador (generalmente P.I.), quien antes de comenzar cada taller explicaba ciertas reglas para mantener un diálogo ágil, ordenado, respetuoso y participativo, fue clave (según observamos y señalaron los participantes). Estas "reglas" incluían estar abiertos a escuchar las opiniones de los demás aunque fueran diferentes a las propias; ser respetuosos con los demás; explicar el uso de términos que otros pudieran desconocer ("jerga científica" y "jerga local"), entre otros. La facilitación de los talleres también aseguraba que todos los participantes o grupos de actores realizaran intervenciones, intentando que éstas fueran balanceadas ( $\sin$ tener actores "dominantes"), y que se analizaran los argumentos planteados por los diferentes grupos antes de llegar a una decisión colectiva. Manejar los conflictos que en ocasiones surgían entre los participantes fue otra desafiante tarea para el equipo facilitador.

En la trayectoria de POPA se pueden reconocer tres "etapas" principales, aunque en cierta forma se superponen en el tiempo y continúan al presente, siendo equivalentes a los ciclos de indagación característicos de una IP. En una primera etapa, el grupo definió una pregunta de investigación sobre la interacción entre la pesca artesanal y los lobos/leones marinos (“¿Cómo es actualmente la interacción de los lobos marinos con el palangre?"), elaborando de forma colectiva un protocolo para utilizar durante muestreos a bordo de las embarcaciones. El palangre (long-line en inglés) es un arte de pesca tradicional en Piriápolis, y los impactos ocasionados por la interacción con los leones marinos son mayores que en la pesca de enmalle debido a la mayor inversión que requiere el palangre (en dinero y tiempo). A pesar de numerosos intentos, el protocolo no llegó a implementarse debido a problemas logísticos (por ej., capacidad limitada de las embarcaciones) y a las bajas expectativas de los pescadores de que dicha investigación contribuiría a solucionar el problema. 
Una segunda etapa fue iniciada concomitantemente con la anterior para atender a otra problemática planteada por los pescadores y de interés para los demás actores: la baja competitividad del pescado artesanal frente a la creciente comercialización en el mercado local del bagre asiático pangasius (Pangasianodon hypophthalmus), el cual en ocasiones es identificado con el nombre de especies de pesca local. POPA decidió trabajar en una estrategia de comunicación para promover una mayor valorización de la pesca artesanal y de sus productos. Como parte de las actividades, y con el apoyo de fuentes nacionales e internacionales, el grupo realizó la Primeria Feria de la Pesca Artesanal en Piriápolis, un festival que duró un fin de semana (febrero de 2012), contó con alrededor de 3000 visitantes, y en el cual los pescadores artesanales, sus artes de pesca y los alimentos derivados de sus capturas, fueron protagonistas (POPA, 2012). Desde entonces POPA cuenta con actividades de comunicación, estando en contacto regular con medios de prensa locales y nacionales. Es importante mencionar el cambio de roles que se experimentó luego de la feria: las autoras dejaron de estar a cargo de la planificación de talleres y de su moderación, tareas que comenzaron a realizar otros integrantes del grupo de forma rotativa. Asimismo, los costos de transporte y alimentación para estas instancias, dejaron de ser cubiertos por los fondos de la investigación doctoral y pasaron a ser cubiertos por apoyos financieros recibidos por POPA. Este cambio de roles de los actores que lideran o coordinan las primeras etapas de una IP es importante para facilitar que el grupo formado pase a auto-gestionarse y a ser autónomo.

En su tercera etapa, POPA dio continuidad a su objetivo de investigar y buscar soluciones a la interacción entre la pesca artesanal y los leones marinos, retomando una idea que había sido planteada por los pescadores en la primera etapa: probar el uso de nasas como arte de pesca alternativo o complementario. Es así que en diciembre de 2012, ante la oportunidad de fondos concursables por parte de la Agencia Nacional de Investigación e Innovación de Uruguay (ANII) y la DINARA, POPA elaboró de forma colectiva, y presentó, un proyecto de investigación titulado "Mitigación del impacto de las interacciones entre leones marinos y pesca artesanal: una investigación participativa para la evaluación de nasas como arte de pesca alternativo". El proyecto fue financiado y en setiembre de 2014, tras haber culminado la etapa de diseño y construcción de las nasas por parte de los integrantes de POPA y técnicos del Laboratorio de Tecnología Pesquera de la DINARA, el proyecto se encuentra en su fase experimental. En esta tercera etapa de POPA, dos de las autoras (M.T. y P.I.) son integrantes del grupo, ya no parte de un "equipo organizador", y realizan diversas tareas en el proyecto.

\section{La investigación participativa en contexto universitario}

Estando las tres autoras vinculadas de alguna manera con la Universidad de la República (UdelaR), consideramos necesario brindar un contexto mínimo para comprender las oportunidades coyunturales asociadas a los tres niveles de enseñanza que se valieron de la IP. La UdelaR ha iniciado en los últimos años un proceso de transformación orientado y definido mediante varios ejes entre los que podemos destacar, en base a los objetivos de este trabajo, la revitalización del ideal latinoamericano de universidad comprometida con la sociedad, abierta, gratuita, cogobernada, democrática en lo interno y orientada a colaborar con la democratización de la sociedad. ${ }^{4}$

Una de las reformas de este período en la UdelaR incluye la creación de un servicio universitario destinado promover actividades interdisciplinarias. El Espacio Interdisciplinario se creó en 2007 y entre sus objetivos principales se encuentra el contribuir a la construcción de estrategias de trabajo universitario que promuevan el abordaje integral de fenómenos y problemas cuya naturaleza desborda los ámbitos disciplinares. Se han apoyado redes temáticas, carreras compartidas y posgrados conjuntos y se ha estimulado el surgimiento y consolidación de nuevos grupos, centros y programas. La tesina de grado (de P.I.) se concretó en el marco de

\footnotetext{
${ }^{4}$ Tomado de un Documento del Rectorado de la UdelaR presentado en marzo de 2008, dirigido a la modificación de la Ley Orgánica de la institución, "Notas para la actualización de la Ley Orgánica de la Universidad de la República”. http://www.universidad.edu.uy/prensa/noticias/ images/imagenes_noticias/RECTORADO_mar08_Notas_para_actualizacion_Ley_Organica.pdf
} 
la Licenciatura en Biología Humana $(\mathrm{LBH})^{5}$, licenciatura que, a pocos años de su creación, fue anidada en el Espacio Interdisciplinario de la UdelaR. La LBH está caracterizada por un plan de estudios flexible e interservicios (interfacultades) que enfrenta al estudiante a diferentes lenguajes, metodologías y puntos de vistas con que son abordados ciertos temas. Dicha flexibilidad propone el desafío, entre otros, de una formación de grado interdisciplinaria (LBH, 2004; Egaña et al., 2010).

Otro de los ejes de las transformaciones universitarias de este último período se relaciona con la promoción de la extensión y la vinculación entre las tres funciones universitarias. Para ello se crearon y consolidaron varias Unidades de Extensión en los distintos servicios de la UdelaR. El Consejo universitario ${ }^{6}$ aprobó un documento relacionado con la enseñanza y la curricularización de la extensión en el que se hace hincapié en el desarrollo armónico e integrado de las tres funciones universitarias en el acto educativo a través de prácticas integrales. La extensión se consideró la función que puede redimensionar los procesos de aprendizaje e investigación al partir de problemas concretos de la sociedad. En este marco se impulsaron Espacios de Formación Integral, prácticas curriculares para la formación de grado que intentan incorporar abordajes interdisciplinarios que favorezcan la articulación de la enseñanza universitaria con la extensión y la investigación. El curso "Actividades Ciencia y Comunidad" (coordinado por M.L.), que trataremos en la sección 4.3 por haber utilizado a la IP, se constituyó en uno de estos espacios de integralidad.

El doctorado (de M.T.) se realizó en la Universidad de Manitoba (Canadá), siendo una propuesta interdisciplinaria vinculada al manejo de recursos naturales y medioambiente. Este tipo de programas interdisciplinarios, sobre todo a nivel de doctorado, son aún escasos en Uruguay.

\subsection{Investigación doctoral e investigación participativa}

El Instituto de Recursos Naturales (NRI) ${ }^{7}$ de la Universidad de Manitoba (Canadá) ofrece dos programas de posgrado interdisciplinarios (maestría y doctorado) en Manejo de recursos naturales y medioambiente (NREM - Natural Resources and Environmental Management). Entre los objetivos de estos programas se destacan el aplicar enfoques holísticos y colaborativos a la solución de problemas, y el vincular la generación de conocimiento con problemas reales para responder a las necesidades sociales existentes y aquellas que van emergiendo (NRI, 2013).

Fue en el marco del doctorado en NREM que M.T. coordinó la organización de la IP en la comunidad pesquera de Piriápolis, en colaboración con la Unidad de Ciencia y Desarrollo (Facultad de Ciencias, UdelaR). La IP en Piriápolis surgió a raíz de uno de los cuatro objetivos específicos de la tesis, el cual consistió en estudiar las contribuciones de las investigaciones participativas en la generación de condiciones necesarias para el co-manejo adaptativo de la pesca artesanal. Para llevar adelante este objetivo, el trabajo de campo fue realizado entre marzo de 2011 y abril de 2012, siendo la observación participante en cada instancia de la IP, y entrevistas semi-estructuradas de forma regular con los participantes, los métodos principales empleados.

A pesar de los muchos desafíos que la literatura señala sobre la realización de tesis doctorales que involucren investigación participativa (ver el análisis crítico de Klocker, 2012), la experiencia en Piriápolis mostró que se trata de iniciativas que pueden ser compatibles. A continuación se analizan cinco desafíos señalados por Klocker (2012) en el contexto de la investigación doctoral e IP en Piriápolis:

(1) Agendas en conflicto. Suele argumentarse que la IP no es compatible con una investigación doctoral ya que antes de iniciar el trabajo de campo hay que tener aprobados el proyecto de tesis, el examen de candidatura y la metodología por el comité de ética. Sin embargo, estos documentos pueden incluir cierta incertidumbre, dejando la puerta abierta para lo que surja durante la IP. En el caso del NRI, algo que evitó mayores dificultades fue que solo uno

\footnotetext{
${ }^{5}$ http://www.lbh.fmed.edu.uy/

${ }^{6}$ Resolución dictada el 27/10/2009 por el Consejo Directivo Central para aprobar el documento "Para la renovación de la enseñanza y la curricularización de la extensión y las actividades en el medio".

${ }^{7} \mathrm{http}$ //umanitoba.ca/institutes/natural_resources/
} 
de los cuatro objetivos específicos de la tesis implicaba IP, siendo los otros tres parte de una investigación convencional.

(2) Falta de apoyo y entrenamiento en IP. Aunque un curso o taller sobre este enfoque como parte de la oferta de cursos del doctorado hubiera sido sumamente enriquecedor, la estudiante compensó esta ausencia realizando una revisión bibliográfica sobre IP y teniendo ricos intercambios con su orientador y comité doctoral antes y durante el desarrollo de la experiencia en Piriápolis.

(3) Individualismo (PhD) vs. Colectivismo (IP). Para compatibilizar la individualidad característica de una investigación doctoral, con el fuerte papel de lo colectivo en una IP, la estudiante tuvo que tener siempre presente la coexistencia entre las actividades del colectivo POPA (el cual ella también pasó a integrar) y sus propias preguntas de investigación que responderían a uno de sus objetivos específicos. Esto fue importante también a la hora de escribir la tesis: la descripción de la IP en Piriápolis fue parte del capítulo sobre métodos, mientras que la evaluación de la IP y el análisis de sus contribuciones al co-manejo constituyó uno de los capítulos de resultados (ver Trimble, 2013). El desarrollo simultáneo de ambas iniciativas (doctorado e IP) fue muy inspirador y motivador, tanto para el desarrollo de los estudios de doctorado como para la continuación de una carrera académica en esa dirección. Si bien es posible de realizar, y propicia muchos aprendizajes, la articulación permanente de los tres roles (estudiante/investigador, organizador/ facilitador y participante) no deja de ser un desafío.

(4) Mayor duración. Suele decirse que no es compatible realizar IP durante un doctorado ya que el último tiene plazos estrictos a ser cumplidos y la IP lleva más tiempo que una investigación convencional. Sin embargo, como argumenta Klocker (2012), los cuatro años de un doctorado proveen de una muy buena oportunidad para estar dedicado a un único proyecto (algo que es más difícil realizar más adelante en la carrera). De forma de respetar los plazos del NRI, la estudiante debió finalizar la etapa de colecta de información para sus preguntas de investigación, a pesar de que la IP de POPA continuaba. El cambio de roles en el grupo propició un aprendizaje personal y también un crecimiento grupal. Sin embargo, aunque los plazos del doctorado se vieron cumplidos y el balance fue positivo, la dedicación fue muy intensa ya que la organización de las actividades de la IP se sumaban a las demás tareas de campo para responder a los otros objetivos de la tesis.

(5) Dudosa validez académica. Los cuestionamientos que suelen hacerse a las IP acerca de su validez, por involucrar a ciudadanos no expertos en el proceso de generación de conocimiento, no surgieron en el NRI ni en los congresos donde este caso fue presentado, pero sí surgió por parte de un revisor de una revista, quien catalogó esta IP como "activismo local apoyado por académicos". El componente de la tesis referido a la IP fue el que todos los examinadores evaluaron más positivamente durante la defensa. Sin embargo, algunos de ellos tuvieron dudas sobre la compatibilidad entre la IP y la tesis doctoral durante la etapa de trabajo de campo, las cuales fueron clarificadas al conocer la evaluación permanente que la estudiante iba realizando del proceso.

\subsection{Pasantía o tesina de grado en el marco de la investigación participativa}

La convocatoria para una pasantía de grado en el marco de la investigación doctoral sobre co-manejo de la pesca artesanal fue realizada en la Facultad de Ciencias (desde la Unidad de Ciencia y Desarrollo), UdelaR. Quien ganara el llamado (P.I.), es estudiante avanzada de la Licenciatura en Biología Humana (LBH), y se involucró con la IP en el contexto antes mencionado. Luego de siete meses de iniciada la IP, la estudiante planteó el objetivo general de la pasantía: analizar la potencialidad de la investigación participativa para abordar 
interdisciplinariamente problemas socio-ambientales que impactan en la salud humana. A lo largo de la pasantía una de las tareas de la estudiante fue facilitar o moderar los talleres grupales, lo cual implicó el desarrollo de ciertas habilidades que propiciaran un diálogo ameno entre los diferentes actores participantes.

A partir de esta experiencia se pueden señalar varias características de esta instancia formativa (tesina de grado en el marco de una IP) que constituyen oportunidades, potencialidades y/o desafíos para una formación de grado interdisciplinaria como propone la LBH:

(1) Al tratarse de un enfoque transdisciplinario, que implicó el abordaje de un problema planteado por la sociedad, la definición del objetivo general de la pasantía presentó algunos retos. En general, al inicio de una pasantía de grado "convencional" se plantea un objetivo y a partir de allí el estudiante comienza su proceso. En el contexto de una IP, uno de los retos que deben asumirse es que el objetivo que va a definir el estudiante para su proyecto de tesis debe vincularse al objetivo, o planteo, del grupo transdisciplinario de trabajo, si se quiere respetar el punto de partida y enfoque de estas investigaciones participativas. En este sentido, la IP es un proceso que requiere una plasticidad tal que permita dialogar a los objetivos académicos con los objetivos del grupo, lo cual puede conllevar más tiempo e incertidumbre, y aumentar el escepticismo desde posturas más academicistas.

(2) La IP permitió acercar dos dimensiones o áreas de conocimiento que suelen estar alejadas académicamente como lo son la biología humana y la acción política. Existen determinantes de la salud humana que son de origen social, político y ambiental (por ej., Galvao et al., 2010), por lo que parece necesario desarrollar estrategias como la IP que aborden desde la acción política las causas de ciertos factores de riesgo socioambiental, los cuales no serían abordables solo desde áreas biológicas del conocimiento.

(3) Esta experiencia permitió conocer e integrar cuáles son las percepciones y/o intereses de los participantes respecto a la pregunta de investigación planteada por la estudiante, a la vez de reflexionar sobre otras inquietudes de los participantes que podrían ser abordadas en futuras instancias.

(4) Permitió ser parte de una plataforma (POPA) que podría posibilitar a futuro el estudio de múltiples problemas de la pesca artesanal desde el campo de la biología humana (por ej., comercialización y consumo de pangasius; posible contaminación en peces de la zona; y temas vinculados a salud pública).

(5) El trabajo transdisciplinario característico de la IP impuso desafíos metodológicos y de lenguajes. Algunos de esos desafíos fueron la aplicación de metodologías provenientes de diferentes disciplinas pero en torno a un objetivo común (lo que implica ciertas traducciones y adaptaciones del lenguaje y por lo tanto habilidades para la comunicación) y la articulación, además, con conocimiento empírico de los pescadores. En este sentido, la IP propició en la estudiante la integración de conocimientos y aprendizajes desde un punto de vista diferente a lo tradicional/disciplinar pues rompió la lógica disciplinaria que a menudo predomina en las propuestas formativas universitarias. La IP generó un espacio donde desarrollar una tesina de grado integral en un ámbito inter/ transdisciplinario.

\subsection{Curso de grado "Actividades Ciencia y Comunidad"}

Todos los planes de estudios de la Facultad de Ciencias-UdelaR (Licenciaturas en ciencias biológicas, bioquímica, geografía, geología, ciencias físicas, matemática) incluyen en su estructura curricular un componente de formación "social y humanística". Esto implica que todos sus estudiantes deben realizar al menos un curso del área social-humanística para completar sus estudios universitarios. En 1994 se creó para ello la Unidad de Ciencia y Desarrollo. Actualmente las opciones son varias pero todas comparten algunos ejes de reflexión común, amparados y nutridos por los enfoques 
Ciencia Tecnología y Sociedad (CTS) ${ }^{8}$, y se centran, con mayor o menor énfasis según el curso, en aspectos éticos, políticos, filosóficos, económicos y sociales de la ciencia. Uno de estos cursos es "Actividades Ciencia y Comunidad", el cual se desarrolla entre la reflexión teórica y la práctica buscando estimular y acompañar las experiencias de interacción y colaboración de los estudiantes con la comunidad. Propicia una enseñanza de "campo" (ciencia-sociedad) que involucra diferentes formas de implicancia ciudadana en el aprendizaje, la comunicación, la generación de conocimiento y la gestión de la ciencia y la tecnología (Lázaro \& Davyt, 2010).

Estas prácticas o actividades se vieron favorecidas por el contexto de transformación universitaria ya mencionado. En la edición 2010 del curso "Actividades Ciencia y Comunidad" se trabajó conjuntamente con la Unidad de Extensión de la Facultad de Ciencias para ofrecer a los estudiantes una diversificación de actividades posibles bajo la órbita de un Espacio de Formación Integral. La actividad debía funcionar como una plataforma de comunicación y actuación bidireccional de aprendizajes colectivos e implicar la puesta en práctica de los saberes propios de las profesiones de la institución. Desde ese año las actividades propuestas se agrupan en distintos ejes de reflexión teórico/práctico para permitir el manejo de contenidos y bibliografía en los encuentros colectivos. Un primer eje agrupa a aquellas actividades vinculadas a la "enseñanza y apropiación social de la ciencia". Otro eje agrupa a las actividades relacionadas con la "participación ciudadana en la generación y gestión del conocimiento", un tercer eje agrupa a actividades vinculadas a las "controversias científico-tecnológicas", y un cuarto eje agrupa a actividades relacionadas con la "inclusión social y conocimiento" (Lázaro \& Davyt, 2010).

Durante el año 2011 una de las actividades propuestas a los estudiantes que optaron por el curso "Actividades Ciencia y Comunidad" fue la de involucrarse en dos experiencias y proyectos de investigación vinculados a pesquerías artesanales que implicaban alguna forma de participación. La actividad se llamó "Problemas en pesquerías artesanales y participación pública: el caso de Piriápolis y Punta del Diablo". Uno de esos proyectos era, precisamente, la IP llevada a cabo en Piriápolis, mientras que el otro proyecto (llamado ROBIN) ${ }^{9}$ se centraba en investigar la dinámica de las poblaciones de especies capturadas en la pesca artesanal de Punta del Diablo (Rocha, Uruguay) y en desarrollar métodos de producción amigables con el ambiente. El programa ROBIN comenzó haciendo énfasis en investigación convencional (por ej., diversidad, dinámica poblacional) incluyéndose luego problemáticas percibidas por los pescadores artesanales (como producción y educación).

Los cinco estudiantes interesados en esta actividad participaron de talleres con los coordinadores de estos dos proyectos y salidas de campo con ambos equipos de trabajo (a Piriápolis y Punta del Diablo), participando además en las actividades de preparación y organización previas. El objetivo principal de la actividad fue generar un ámbito teórico/práctico de análisis y reflexión sobre la participación de distintos actores en proyectos de investigación, comparando ambas iniciativas. El involucramiento de estos estudiantes de ciencias fue durante una etapa temprana de la IP en Piriápolis (setiembre-octubre 2011), lo que dificultó que identificaran el componente más claro de "investigación" (tener preguntas de investigación y una metodología para responderlas), pero permitió que conocieran de primera mano algunas condiciones necesarias de la implicancia temprana de otros actores para identificar y diagnosticar problemáticas reales. Los estudiantes resaltaron en varias oportunidades el hecho de que todos los actores participantes hacían aportes y, a pesar de diferentes visiones, se llegaban a acuerdos de trabajo. Se interesaron especialmente en analizar las diferencias entre el tipo de participación comunitaria de los dos proyectos, y sobre la justificación del "momento" adecuado para la participación de

\footnotetext{
${ }^{8}$ Las dimensiones sociales de la ciencia y la tecnología tanto en sus antecedentes y condicionantes como en sus consecuencias sociales y ambientales, son el objeto más general de este enfoque CTS que desde la década del 70 del siglo pasado promueve una renovación de los contenidos educativos basada en la incorporación de los contextos sociales en las prácticas vinculadas a la enseñanza de las ciencias. La enseñanza CTS estimula el contacto entre las culturas humanística y científico-tecnológica; el espíritu crítico y el sentido de responsabilidad en el estudiante, así como la reflexión sobre la democratización de la ciencia y la tecnología (Lázaro \& Davyt, 2010).

${ }^{9}$ http://robinpuntadeldiablo.blogspot.com/
} 
los “otros" implicados (no científicos) según el tipo de problema o la investigación.

Una de las dificultades de este tipo de práctica para los estudiantes a nivel de grado es tratar de combinar el tiempo de desarrollo de la IP con los tiempos curriculares. Una IP (o cualquier actividad que implique otros actores y problemas complejos para "investigar") no puede adaptarse fácilmente a un calendario semestral. De todas formas, una IP puede servir de paraguas para una "visita" por el enfoque y la práctica, manejando algunos objetivos específicos vinculados a los contenidos del curso. Como desafío también se puede mencionar que "Actividades Ciencia y Comunidad" ha sido el curso menos elegido por parte de los estudiantes debido posiblemente, además de la razón anterior, a apartarse de prácticas más tradicionales desde el punto de vista de la enseñanza, que no requieren de tanto protagonismo por parte de los estudiantes, ni manejan tanta incertidumbre sobre los resultados (propio del trabajo con "otros"). La falta de costumbre y el incentivo a transitar por las carreras en el menor tiempo posible pueden atentar contra la elección de este curso optativo.

La visión de este tipo de experiencia como una práctica de extensión "tradicional" 10 (disponer un tiempo voluntario, y extra, en su formación, para "devolver" a la sociedad algo de lo que le da en apoyo a la universidad) y no necesariamente como una práctica justificada por la propia situación de las ciencias ambientales y del trabajo de investigador (elemento en el que enfatiza el enfoque CTS para la enseñanza de la ciencia) es común en los estudiantes. En las reuniones teóricas del curso se intenta reflexionar sobre ello y vincularlo con las concepciones de ciencia detrás de los distintos tipos de prácticas (como los elementos detallados en la introducción de este trabajo).

\section{Discusión y conclusiones}

Las investigaciones participativas en las que académicos, comunitarios y otros actores, trabajan co- laborativamente como co-investigadores a lo largo de un proyecto de investigación que definen de forma conjunta, representan una valiosa estrategia para el análisis, comprensión y acción frente a problemas ambientales locales (Blackstock et al., 2007; Wilmsen et al., 2008; Wiber et $a l ., 2009)$. Es preciso señalar que las IP representan una herramienta entre otras y no una que pretende reemplazar a las investigaciones convencionales o erigirse como estrategia única. Son especialmente adecuadas en el contexto de problemas ambientales, incluyendo los referentes al manejo de recursos naturales (donde también está habiendo una transición a modos participativos de toma de decisiones), debido a los diversos resultados positivos que conllevan, como la co-producción de conocimiento, el aprendizaje social y una mejora en las relaciones entre los actores participantes, entre otros (Blackstock et al., 2007; Trimble \& Berkes, 2013; Trimble \& Lázaro, 2014).

Las experiencias formativas detalladas en este trabajo mostraron que el enfoque de investigación participativa es compatible con la realización de tesinas de grado y tesis de posgrado, así como adaptables algunos de sus componentes a cursos de grado. Una investigación doctoral puede incluir IP en alguno(s) de sus objetivo(s), como en el caso que analizamos, o puede consistir enteramente de IP (Klocker, 2012). En el caso de programas de maestría (aunque no estuvieron comprendidos en nuestro análisis), podemos sugerir que dada su duración (dos años en promedio), sería conveniente que los tesistas realizaran su investigación en el marco de un proyecto mayor (como fuera el caso de la estudiante de la LBH), en vez de iniciar ellos mismos la IP. Así como planteamos que este enfoque no busca reemplazar a las investigaciones convencionales, es de subrayar que la vinculación de tesistas de grado y posgrado a investigaciones participativas dependerá de sus intereses y de sus potenciales objetivos (no siendo recomendable para todos los casos).

Si bien las tres experiencias de formación universitaria descritas en este trabajo, vinculadas a la IP en la pesquería artesanal de Piriápolis, fueron muy enriquecedoras (sus ventajas o potencialidades se retoman

\footnotetext{
${ }^{10}$ La extensión, como función universitaria típica del modelo latinoamericano, se propuso en tiempos de combate al elitismo y como arma de transformación de la sociedad, sin necesariamente cuestionar o reconocer la interdependencia de la ciencia con su contexto social. Actualmente, la extensión interpela la noción frecuente de que el poder del conocimiento esté concentrado en manos expertas y enfatiza en el diálogo bidireccional del vínculo entre la universidad y la sociedad (Lázaro \& Davyt, 2010).
} 
a continuación), las mismas forman parte de un grupo de oportunidades de formación interdisciplinaria aún escasas en Uruguay, a pesar de los procesos de transformación universitaria. ${ }^{11}$ El curso "Actividades Ciencia y Comunidad" (Facultad de Ciencias, UdelaR) es optativo y no tiene una matrícula importante de estudiantes; la Licenciatura en Biología Humana (LBH, UdelaR) permite la realización de tesinas interdisciplinares pero predominan los enfoques disciplinares; y el Doctorado en Manejo de Recursos Naturales y Medioambiente (Universidad de Manitoba) exige la realización de una tesis interdisciplinaria pero no necesariamente la inclusión de un enfoque participativo de investigación. Estas tres experiencias formativas (curso, licenciatura, doctorado), que tienen en común el trascender la formación disciplinar, utilizaron plataformas que posibilitaron el desarrollo y vinculación con una IP. La existencia de más de estos espacios que faciliten y procuren la inter y transdisciplina, así como la integralidad de funciones universitarias, es un elemento clave para propiciar la puesta en marcha de investigaciones participativas. A su vez, el desarrollo de más IP vinculadas a la universidad podría propiciar la oferta de más cursos de esta índole (como el Espacio de Formación Integral "IP aplicada a las ciencias ambientales" iniciado en 2014 en la UdelaR).

Las tres experiencias permiten extraer algunas conclusiones sobre las IP en contextos de enseñanza universitaria. Un curso como "Actividades Ciencia y Comunidad", en el que se intenta practicar el vínculo ciencia-sociedad y reflexionar sobre ello, es una plataforma idónea para tratar de integrar investigación con extensión en un contexto de aprendizaje colaborativo. Pero también podría utilizarse el enfoque de IP en otros cursos vinculados específicamente a las ciencias ambientales. El desafío es, como se decía anteriormente, que este tipo de investigación se conciba como una opción válida. Eso requiere de cambios en las prácticas y también cambios en las concepciones de la investigación y metodología científica. Los enfoques positivistas sobre la actividad científica siguen campeando en el mundo universitario.

Los procesos interdisciplinarios y transdisciplinarios implican la convergencia de conocimientos provenientes de diferentes disciplinas y/o actores, y van más allá de la sumatoria o simple yuxtaposición de los mismos (Olivé, 2011). Si nos ubicamos en una carrera que desde el inicio se propone la interdisciplinariedad como norma, como lo es la $\mathrm{LBH}$, cabe hacer las siguientes apreciaciones. Si bien un estudiante de la LBH a partir de su tránsito interservicios adquiere a lo largo de su carrera ciertas habilidades, tales como la comprensión de diferentes lenguajes académicos y diferentes herramientas metodológicas, poner en práctica y fortalecer dichas habilidades implica trabajar bajo ciertas estrategias que permitan un diálogo activo para lograr la conexión e integración entre los diferentes saberes y puntos de vista. La IP brindó una plataforma desde donde estimular, aprender y practicar la inter y transdisciplina, contribuyendo no solo a la formación de la estudiante sino a los espacios de reflexión académica de la propia licenciatura. Por otra parte, la IP acercó dos ámbitos que habitualmente se consideran aislados: la biología humana y la acción política. Este acercamiento permitió la reflexión sobre la importancia de desarrollar espacios de participación en temas que vinculan la salud humana con los problemas ambientales y el manejo de recursos naturales, ámbitos políticos por excelencia. Alumbrar este tipo de vínculos existentes entre la biología humana y los problemas ambientales puede, a través de la participación, acercar a otros actores implicados que están ausentes en ciertas discusiones.

La experiencia de doctorado sugiere que el proceso de una tesis doctoral, que implica producir conocimiento nuevo y aportar al desarrollo de los conceptos o teorías utilizados, puede compatibilizarse con un espacio participativo de diagnóstico, investigación y resolución de problemas. Un tema de tesis vinculado a conflictos socio-ambientales (y específicamente recursos naturales), como son los vinculados con el posgrado en cuestión, implica ineludiblemente múltiples actores (con sus respectivos intereses, saberes, etc.), y por lo tanto la necesidad de generar espacios que propicien la formación de relaciones de confianza y el aprendizaje colectivo. De hecho, una de las preguntas de investigación de la tesis doctoral refería a analizar los aprendizajes que

\footnotetext{
${ }^{11}$ En lo que refiere al manejo de recursos naturales se puede mencionar, por ejemplo, la Maestría en Manejo Costero Integrado, también en el ámbito del reciente Espacio Interdisciplinario.
} 
experimentaban los participantes de la IP y los cambios en las relaciones entre ellos, considerando que ambos son condiciones para un futuro co-manejo de la pesca artesanal. La estudiante aprendió sobre cómo poner en práctica este enfoque participativo de investigación y sobre su vínculo con ámbitos de manejo pesquero.

El ahora ya consolidado Grupo POPA representa una plataforma en sí misma para futuros proyectos de investigación participativa (como la actual iniciativa sobre un nuevo arte de pesca en Piriápolis: las nasas), existiendo la oportunidad de vincularse con espacios de enseñanza universitaria en diversas carreras. En particular, la tesis de una integrante de POPA, estudiante de la Maestría en Comunicación y Cultura de la Universidad Católica del Uruguay, está enfocada en los resultados que tuvo la Primera Feria de la Pesca Artesanal en Piriápolis en relación a la valorización del pescador artesanal.

A pesar de estas ventajas y oportunidades de aprendizaje que pueden brindar las IP, y la necesidad de asumir los desafíos que implica transitar hacia un mayor desarrollo de investigaciones participativas (conceptuales, pedagógicos, epistemológicos), hay también que estudiar formas de sortear algunos problemas prácticos como los mencionados en relación a las tres experiencias. Posiblemente el mayor de ellos es el tiempo insumido por la investigación participativa (por ej., la etapa de trabajo de campo para la tesina de grado y para la tesis de doctorado fueron más largas que en promedio), lo cual podría llegar a dificultar el cumplimiento de los tiempos curriculares.

\section{Referencias}

Ander-Egg, E. Interdisciplinariedad en educación. Buenos Aires: Editorial Magisterio del Río de la Plata, 1994.

Arnold, J. S.; Fernandez-Gimenez, M. Building Social Capital Through Participatory Research: An Analysis of Collaboration on Tohono O'odham Tribal Rangelands in Arizona. Society and Natural Resources, 20, 481-495, 2007.

Balcazar, F. Investigación acción participativa (IAP): aspectos conceptuales y dificultades de implementación. Fundamentos en humanidades. Universidad Nacional de San Luis, IV, I/II (7/8), 59-77, 2003.
A modo de reflexiones finales, el análisis de las tres experiencias formativas vinculadas a la IP en Piriápolis (Uruguay) sugiere que para un mayor uso de este enfoque desde el ámbito universitario, es necesaria la existencia de espacios que propicien y justifiquen la participación de los "otros" (actores implicados no académicos) en el proceso de diagnóstico, investigación y búsqueda de soluciones a los problemas que los afectan. Para ello se requiere de una reflexión mayor sobre temas más generales vinculados a las concepciones de ciencia, a su rol social y a la democratización del conocimiento. Una forma de propiciar esta reflexión puede ser la inclusión de cursos o módulos CTS (Ciencia Tecnología y Sociedad), del tipo de "Actividades Ciencia y Comunidad" en distintos momentos de las carreras de grado y posgrado. Asimismo, tiene que haber flexibilidad, a nivel de las estructuras docentes y académicas, para propiciar el acercamiento entre la investigación y problemáticas locales, así como entre diferentes disciplinas y diferentes saberes. Acompañado de la oferta de recursos financieros para las IP, es preciso que aumente el reconocimiento de los académicos a los aportes que pueden realizar diferentes actores a una mejor comprensión de los problemas que se estén abordando, lo cual es una característica del hacer "ciencia con la gente" (y no "para la gente") en un contexto posnormal (Funtowicz \& Ravetz, 2000). La evaluación sistemática de investigaciones participativas permitirá brindar evidencia empírica acerca de los resultados positivos de esta estrategia.

Blackstock, K. L.; Kelly, G. J.; Horsey, B. L. Developing and applying a framework to evaluate participatory research for sustainability. Ecological Economics, 60 (4), 726-42, 2007.

Brzeski, V.; Graham, J.; Newkirk, G. Participatory Research and CBCRM: In Context. Nova Scotia: Coastal Resources Research Network, Dalhousie University, Halifax; Ottawa: International Development Research Centre, 2001. Disponible en: http:// idl-bnc.idrc.ca/dspace/bitstream/10625/46513/1/132987.pdf.

Cambell J.; Salagrama. V. New approaches to participation in fisheries research. Rome: FAO Fisheries Circular No.965, 2001. 
Chambers, R. The origins and practice of participatory rural appraisal. World Development, 22(7), 953-969, 1994.

Chevalier J. M; Buckles, D. J. Participatory action research: theory and methods for engaged inquiry. London and New York: Routledge, 2013.

Conway, F. D. L.; Pomeroy, C. Evaluating the human - as well as the biological - objectives of cooperative fisheries research. Fisheries, 31(9), 447-454, 2006.

Cornwall, A.; Jewkes, R. What is participatory research? Social Science and Medicine, 41(12), 1667-1676, 1995.

de Sousa Santos, B. La Universidad en el siglo XXI. Para una reforma democrática y emancipatoria de la universidad. Montevideo: Universidad de la República-Ediciones Trilce, 2010.

Egaña, A.; Bardier C.; Botto G.; González L.; Sans M. La trayectoria curricular del estudiante de biología humana como formación para la interdisciplina. En: En_clave inter 2010. Reflexiones sobre la interdisciplina en la Universidad de la República. Montevideo: Zonalibro S.A, 2010.

Fals Borda, O. The application of participatory action-research in Latin America. International Sociology, 2(4), 329-347, 1987.

Fals Borda, O. The North-South convergence: A 30-year firstperson assessment of PAR. Action Research, 4(3), 351-358, 2006.

Fals Borda, O.; Rahman, M. A. Action and Knowledge: Breaking the Monopoly with Participatory Action Research. Nueva York: Apex Press, 1991.

Fiorino, D. J. Citizen Participation and Environmental Risk: A Survey of Institutional Mechanisms. Science, Technology, and Human Values, 15(2), 226-243, 1990.

Fisher, F. Citizens, Experts, and the Environment: The politics of local knowledge. Londres: Duke University Press, 2000.

Freire, P. Pedagogía del oprimido. Buenos Aires: Siglo XXI Editores Argentina S.A., 2. ed., 1970.

Funtowicz, S. O.; Ravetz, J. R. La ciencia posnormal: ciencia con la gente. Barcelona: Icaria, 2000.

Galvao, L.; Finkelman, J.; Henao, S. (Eds.) Determinantes ambientales y sociales de la salud. México: McGraw-Hill Interamericana Editores S.A de C.V., 2010.

Gaventa, J.; Cornwall, A. Power and knowledge. In: Reason. P.; Bradbury, H. (Eds). The Sage Handbook of Action Research: Participative Inquiry and Practice. London: Sage Publications, p. 70-80, 2001.
Gonsalves, J.; Becker, T.; Braun, A.; Campilan, D.; De Chavez, H.; Fajber, E.; Kapiriri, M.; Rivaca-Caminade, J.; Vernooy, R. (Eds). Investigación y Desarrollo Participativo para la Agricultura y el Manejo Sostenible de Recursos Naturales: Libro de Consulta. Volumen 1: Comprendiendo. Investigación y Desarrollo Participativo. Perspectivas de los Usuarios con la Investigación y el Desarrollo Agrícola. Ottawa: Centro Internacional de la Papa, Laguna, Filipinas y Centro Internacional de Investigaciones para el Desarrollo, 2006. Disponible en: http:// web.idrc.ca/es/ev-73443-201-1-DO_TOPIC.html.

Hall, B. L. From margins to center? The development and purpose of participatory research. The American Sociologist, 23(4), 15-28, 1992.

Hartley, T. W.; Robertson. R. A. Stakeholder engagement, cooperative fisheries research and democratic science: the case of the Northeast Consortium. Human Ecology Review, 13(2), 161-71, 2006.

Holling, C. S. Simplifying the complex: the paradigms of ecological function and structure. European Journal of $\mathrm{Op}$ erational Research, 30, 139-146, 1987.

Jacobsen, R. B.; Wilson, D. C. K.; Ramirez-Monsalve, P. Empowerment and regulation - dilemmas in participatory fisheries science. Fish and Fisheries, 13, 291-302, 2012.

Johnson, T. R. Cooperative research and knowledge flow in the marine commons: Lessons from the Northeast United States. International Journal of the Commons, 4(1), 251-72, 2010.

Johnson, T. R.; van Densen, W. L. T. Benefits and organization of cooperative research for fisheries management. ICES Journal of Marine Science, 64(4), 834-840, 2007.

Kaplan, I. M.; McCay. B. J. Cooperative research, co-management and the social dimension of fisheries science and management. Marine Policy, 28(3), 257-258, 2004.

Kemmis, S.; McTaggart. R. Participatory Action Research: Communicative Action and the Public Sphere. In: Denzin, N. K.; Lincoln, Y. S. (Eds). The Sage Handbook of Qualitative Research. Thousand Oaks, California: Sage Publications, p. 559-603, 2005.

Kindon, S. Participatory Action Research. In: Hay, I. (Ed). Qualitative Research Methods in Human Geography. Melbourne: Oxford University Press, p. 207-220, 2008.

Klein, J. T. An interdisciplinary lexicon. In: Interdisciplinarity: History, Theory and Practice. Wayne State University Press, p. 55-73, 1990 . 
Klocker, N. Doing Participatory Action Research and Doing a $\mathrm{PhD}$ : Words of Encouragement for Prospective Students. Journal of Geography in Higher Education, 36(1), 149-163, 2012.

Lang, D. J.; Wiek, A.; Bergmann, M.; Stauffacher, M.; Martens, P.; Moll, P.; Swilling, M.; Thomas, C. J. Transdisciplinary research in sustainability science: practice, principles, and challenges. Sustainability Science, 7(1), 25-43, 2012.

Lázaro, M.; Davyt, A. La enseñanza CTS y la integración de las funciones universitarias: reflexiones desde una facultad de ciencias. Redes, 16(31), 145-161, 2010.

Lázaro, M.; Trimble, M.; Umpiérrez, A.; Vasquez, A.; Pereira, G. Juicios Ciudadanos en Uruguay: dos experiencias de participación pública deliberativa en ciencia y tecnología. Montevideo-Uruguay. 2013. Disponible en: http://www.juiciociudadano.org/libro/.

LBH (Licenciatura en Biología Humana). Detalles de la carrera. 2004. Disponible en: http://www.lbh.fmed.edu.uy/ Detalles_de_la_Carrera.pdf. Consultado en: ene. 2014.

Lewin, K. Action research and minority problems. Journal of Social Issues, 2, 34-36, 1946.

NRI - Natural Resources Institute. NRI Graduate Student Handbook. University of Manitoba, Winnipeg, 2013.

Olivé, L. Interdisciplina y transdisciplina desde la filosofía. Ludus Vitalis, XIX(35), 251-256, 2011.

POPA - Por la Pesca Artesanal en Piriápolis. Informe de la Primera Feria de la Pesca Artesanal en Piriápolis. Grupo Por la Pesca Artesanal, Piriápolis, 2012.

Reason, P. Three approaches to participatory inquiry. In: Denzin, N. K.; Lincoln, Y. S. (Eds). Handbook of Qualitative Research. Londres: Sage, p. 324-339, 1994.

Rowe, G.; Frewer, L. J. Public participation methods: A framework for evaluation. Science Technology and Human Values, 25(1), 3-29, 2000.

Shirk, J. L.; Ballard, H. L.; Wilderman, C. C.; Phillips, T.; Wiggins, A.; Jordan, R.; McCallie, E.; Minarchek, M.; Lewens- tein, B. V.; Krasny, M. E.; Bonney, R. Public participation in scientific research: a framework for deliberate design. Ecology and Society, 17(2), 29, 2012. http://www.ecologyandsociety. org/vol17/iss2/art29/

Stephens, J. B.; Berner, M. Learning from your neighbor: The value of public participation evaluation for public policy dispute resolution. Journal of Public Deliberation, 7(1), 2011.

Trimble, M. Towards adaptive co-management of artisanal fisheries in coastal Uruguay: analysis of barriers and opportunities, with comparisons to Paraty (Brazil). Winnipeg, Tesis (Doctorado en Manejo de Recursos Naturales y Medio Ambiente) - University of Manitoba, 2013. Disponible en: https://umanitoba.ca/institutes/natural_resources/canadaresearchchair/thesis/trimble_micaela.pdf

Trimble, M.; Berkes, F. Participatory research towards co-management: Lessons from artisanal fisheries in coastal Uruguay. Journal of Environmental Management, 128, 768-778, 2013.

Trimble, M.; Johnson, D. Artisanal fishing as an undesirable way of life? The implications for governance of fishers' wellbeing aspirations in coastal Uruguay and southeastern Brazil. Marine Policy, 37, 37-44, 2013.

Trimble, M.; Lázaro, M. Evaluation criteria for Participatory Research: Insights from coastal Uruguay. Environmental Management, 54, 122-137, 2014.

Wiber, M.; Berkes, F.; Charles, A.; Kearney, J. Participatory research supporting community-based fishery management. Marine Policy, 28(6), 459-68, 2004.

Wiber, M.; Charles, A.; Kearney, J.; Berkes, F. Enhancing community empowerment through participatory fisheries research. Marine Policy, 33(1), 172-179, 2009.

Wilmsen, C.; Elmendorf, W.; Fisher, L.; Ross, J.; Sararthy, B.; Wells, G. Partnerships for empowerment: participatory research for community-based natural resource management. London: Earthscan, 2008. 